\title{
Numerical Simulations of Flow Separation Control in Low- Pressure Turbines using Plasma Actuators
}

\author{
Y. B. Suzen* \\ North Dakota State University, Fargo, ND 58105 \\ P. G. Huang ${ }^{\dagger}$ \\ Wright State University, Dayton, $\mathrm{OH} 45435$ \\ D. E. Ashpis \\ NASA John H. Glenn Research Center at Lewis Field, Cleveland, OH 44135
}

\begin{abstract}
A recently introduced phenomenological model to simulate flow control applications using plasma actuators has been further developed and improved in order to expand its use to complicated actuator geometries. The new modeling approach eliminates the requirement of an empirical charge density distribution shape by using the embedded electrode as a source for the charge density. The resulting model is validated against a flat plate experiment with quiescent environment. The modeling approach incorporates the effect of the plasma actuators on the external flow into Navier Stokes computations as a body force vector which is obtained as a product of the net charge density and the electric field. The model solves the Maxwell equation to obtain the electric field due to the applied AC voltage at the electrodes and an additional equation for the charge density distribution representing the plasma density. The new modeling approach solves the charge density equation in the computational domain assuming the embedded electrode as a source therefore automatically generating a charge density distribution on the surface exposed to the flow similar to that observed in the experiments without explicitly specifying an empirical distribution. The model is validated against a flat plate experiment with quiescent environment.
\end{abstract}

\section{Nomenclature}

$\begin{array}{ll}\vec{E} & =\text { electric field, N/C } \\ \varepsilon & =\text { permittivity, } \varepsilon=\varepsilon_{r} \varepsilon_{o} \\ \varepsilon_{r} & =\text { relative permittivity } \\ \varepsilon_{o} & =\text { permittivity of free space, } 8.854 \times 10^{-12} \mathrm{C}^{2} / \mathrm{Nm}^{2} \\ \vec{f}_{B} & =\text { body force vector, } \mathrm{N} / \mathrm{m}^{3} \\ \Phi & =\text { total electric potential, Volt, } \Phi=\phi+\varphi \\ \phi & =\text { electric potential due to external electric field, Volt } \\ \varphi & =\text { electric potential due to net charge density, Volt } \\ L_{e} & =\text { length of the electrode } \\ \lambda_{d} & =\text { Debye length, m } \\ \mu & =\text { location parameter for Gaussian distribution } \\ \omega & =\text { frequency, Hz } \\ \operatorname{Re} & =\text { Reynolds number based on inlet velocity and axial chord } \\ \rho_{c} & =\text { net charge density, C/m }\end{array}$

\footnotetext{
*Assistant Professor, Dept. of Mechanical Engineering and Applied Mechanics, 108 Dolve Hall, bora.suzen@ndsu.edu, Senior Member AIAA.

†Professor and Chair, Dept. of Mechanical and Materials Engineering, 3640 Colonel Glenn Hwy., george.huang@wright.edu, Senior Member AIAA.

Aerospace Engineer, Turbine Branch, Mail Stop 5-11, ashpis@ nasa.gov, Senior Member AIAA.
} 


$\begin{array}{ll}t & =\text { time, } \mathrm{s} \\ \mathrm{U}_{\mathrm{in}} & =\text { inlet velocity } \\ \mathrm{U} & =\text { streamwise velocity } \\ x, y & =\text { coordinates } \\ \mathrm{y}_{\mathrm{n}} & =\text { wall normal distance }\end{array}$

\section{Introduction}

$\mathrm{P}$ asma actuators have been shown to be effective in several flow-control-related applications including flow separation and boundary layer control. The plasma actuators consist of two electrodes that are located on a surface separated by a dielectric material as shown in Fig. 1. A high-voltage AC supplied to the electrodes causes the air in their vicinity to weakly ionize. The ionized air (plasma), in the presence of the electric field gradient produced by the electrodes, result in a body force vector acting on the external flow that can induce steady or unsteady velocity components. The effectiveness of plasma actuators in controlling flow separation has been demonstrated by several researchers [1-9]. These experiments showed that a range of parameters have to be taken into consideration for effective flow control including the location of the actuators on the surface, orientation, size, and relative placement of the embedded and exposed electrodes, applied voltage, and frequency of the actuation. Due to a large number of parameters involved, optimizing the performance of actual applications can be a fairly complicated task. CFD simulations can provide a useful tool in design and optimization of such complex flow control systems.

Computational studies of plasma flow control have been limited in comparison to the vast number of experimental studies reported [10-14]. Recently, the authors developed a simple phenomenological numerical simulation methodology for active flow separation control applications using plasma actuators [15]. In this new approach, the effect of the plasma actuators on the external flow is incorporated into Navier Stokes computations as a body force vector. The body force is obtained as a product of the net charge density and the electric field. The new model solves the Maxwell equation to obtain the electric field due to the applied AC voltage at the electrodes and an additional equation for the charge density representing the plasma density. However, there was an assumption made to the boundary condition of the charge density on the surface of the above the embedded electrode and this makes the extension of the model to complex and/or 3D flows impossible. This paper is an attempt to remedy this weakness. The model is summarized in the next section without the readers having to refer to our previous paper.

\section{Modeling Plasma Actuator Physics in CFD Computations}

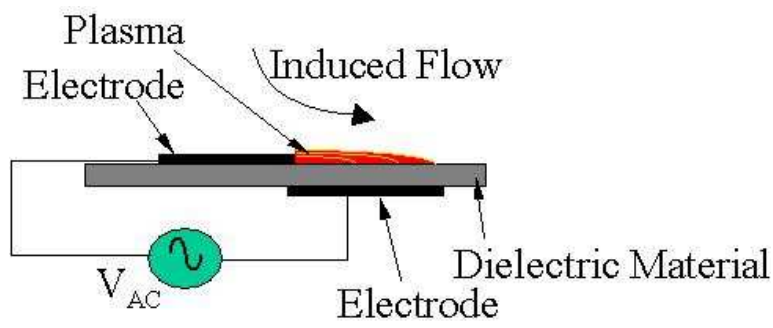

Figure 1. Schematic of plasma actuator.

The body force that the plasma actuator induces on the external flow can be expressed in terms of the applied voltage and incorporated into the Navier Stokes equations. By neglecting magnetic forces, the electrohydrodynamic (EHD) force can be expressed as

$$
\vec{f}_{B}=\rho_{c} \vec{E}
$$

where, $\vec{f}_{B}$ is the body force per unit volume, $\rho_{c}$ is net the charge density and $\vec{E}$ is the electric field. If the time variation of the magnetic field is negligible, as is often the case in plasma, the Maxwell's equations give rise to $\nabla \times E \approx 0$. This implies that the electric field can be derived from the gradient of a scalar potential[16]:

$$
\vec{E}=-\nabla \Phi \text {. }
$$

Gauss's law yields:

$$
\nabla \cdot(\varepsilon \vec{E})=\rho_{c}
$$




$$
\nabla \cdot(\varepsilon \nabla \Phi)=-\rho_{c}
$$

where $\varepsilon$ is the permittivity. The permittivity can be expressed as:

$$
\varepsilon=\varepsilon_{r} \varepsilon_{o}
$$

where $\varepsilon_{r}$ is the relative permittivity of the medium, and $\varepsilon_{o}$ is the permittivity of free space.

If we write the net charge density within the plasma at any point in terms of the elementary charge $e$, the background plasma density $n_{o}$, the temperature of the species $T$, and introduce the Debye length $\lambda_{d}$ the charge density can be expressed in terms of the potential $\Phi$ and the Debye length $\lambda_{d}[16]$,

$$
\rho_{c} / \varepsilon_{o}=\left(-1 / \lambda_{d}^{2}\right) \Phi
$$

The body force given by Eq. (1) can be calculated using Eqs. (2) and (6) by imposing the applied voltage at the electrodes as boundary conditions.

In the current modeling approach the electric potential is decomposed into two parts by using the superposition technique [15]. Since the gas particles are weakly ionized, we can assume the potential $\Phi$ can be decoupled into two parts: one being a potential due to the external electric field, $\phi$, and the other being a potential due to the net charge density in the plasma, $\varphi$,

$$
\Phi=\phi+\varphi
$$

This approach is similar to the one used in numerical simulation of electroosmotic flows in which case the external electric field generates a force on the charged particles creating flow[17].

If we assume that the Debye thickness is small and the charge on the wall is not large, the distribution of charged species in the domain is governed by the potential caused by the electric charge on the wall and is largely unaffected by the external electric field. Therefore, we can write two independent equations in terms of these two potentials, one for the external electric field due to the applied voltage at the electrodes:

$$
\nabla \cdot\left(\varepsilon_{r} \nabla \phi\right)=0
$$

and another one for the potential due to the charged particles:

$$
\nabla \cdot\left(\varepsilon_{r} \nabla \varphi\right)=-\left(\rho_{c} / \varepsilon_{o}\right) .
$$

Using $\rho_{c} / \varepsilon_{o}=\left(-1 / \lambda_{d}^{2}\right) \varphi$ Eq. (9) can be written as[16],

$$
\nabla \cdot\left(\varepsilon_{r} \nabla \rho_{c}\right)=\rho_{c} / \lambda_{d}^{2} .
$$

Eq. (8) provides the solution for the electric potential, $\phi$, using the applied voltage on the electrodes as boundary conditions and $\rho_{c}$ is obtained from Eq. (10) with a prescribed boundary condition on the surface over the embedded electrode. The boundary condition is synchronized with the applied voltage on the electrode and the resultant body force vector is computed by

$$
\vec{f}_{B}=\rho_{c} \vec{E}=\rho_{c}(-\nabla \phi)
$$

The boundary conditions and the computational domains of Eqs. (8) and (10) for a single pair of electrodes are shown in Figures $2 \mathrm{a}$ and $2 \mathrm{~b}$, respectively. Equation (8) is solved for the electric potential, $\phi$, imposing the applied voltage on the electrodes as boundary conditions and using the appropriate $\varepsilon_{r}$ value on both the air side and the wall. For air, $\varepsilon_{r}=1.0$ and the experiments we considered for test cases used Kapton as the dielectric material which has an $\varepsilon_{r}$ value of 2.7. On the wall-air interface harmonic mean of $\varepsilon_{r 1}$ and $\varepsilon_{r 2}$ must be used in order to conserve electric field[15]. 


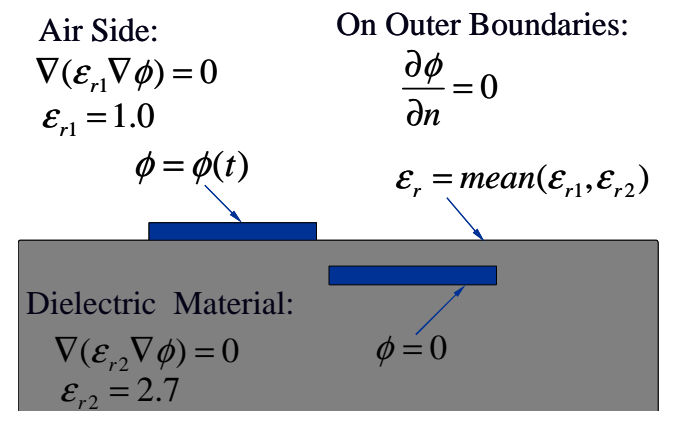

(a)Boundary conditions for Eq. (8)

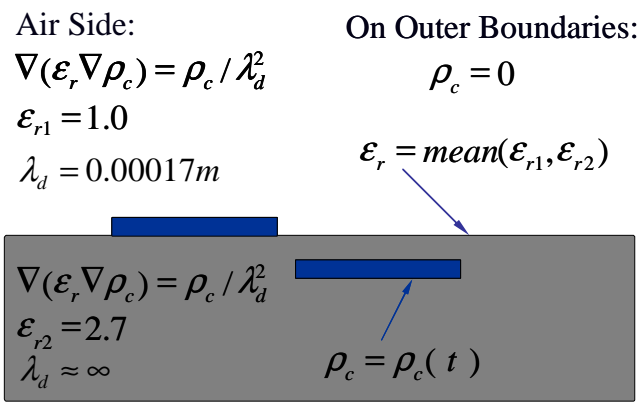

(b) Boundary conditions for Eq. (10) Figure 2. Boundary conditions and computational domain for Eqs. (8) and (10).

Applied AC voltage imposed at the exposed (upper) electrode as boundary condition is:

$$
\phi(t)=\phi^{\max } f(t)
$$

The wave form function, $f(t)$, can be a sine wave given by:

$$
f(t)=\sin (2 \pi \omega t)
$$

where $\omega$ is the frequency and $\phi^{\max }$ is the amplitude, both of which are known quantities from experiment. The embedded electrode is prescribed as ground by setting the electric potential to zero on that electrode. At the outer boundaries, $\partial \phi / \partial n=0$ is assumed as shown in Figure $2 \mathrm{a}$.

In order to obtain a net charge density distribution over the embedded electrode resembling the plasma distribution observed in experiments $[16,18,19]$, we introduce a new approach which eliminates the need for prescribing a charge density distribution on the surface over the embedded electrode. In this new method, Eq. (10) is solved assuming the lower electrode as a source for the net charge density, $\rho_{c}$, which is synchronized with the time variation of the applied voltage $\phi(t)$ on the exposed electrode, $f(t)$ in equation (12):

$$
\rho_{c}(t)=\rho_{c}^{\max } f(t)
$$

where $\rho_{c}^{\max }$ is the maximum value of the charge density allowed in the domain (in Coulomb $/ \mathrm{m}^{3}$ ) and is a parameter to be determined later. By assuming the embedded electrode as a source for the charge density, solution of Eq. (10) automatically results in a charge density distribution on the surface above the embedded electrode similar to the distribution observed in experiments and the model does not require prescription of a charge density distribution on the surface and thus makes the modeling of generic actuator geometries including generic shapes and orientations of electrodes straight forward.

At this stage, it should be noted that in order to solve Eq. (10) we need to specify two parameters, namely, $\rho_{c}^{\max }$ and $\lambda_{d}$. These parameters control the strength of the plasma actuator's effects on the flowfield and extent of these effects into the flowfield. The Debye length, $\lambda_{d}$, is in the order of $0.00017 \mathrm{~m}$ for the case considered here. The maximum charge density, $\rho_{c}^{\max }$, is a parameter to be calibrated using available experimental data.

It is important to note that, Eqs. (8) and (10) need to be solved only once at the beginning of Navier Stokes computations since these equations do not contain a time derivative term. This can be achieved by writing these equations in nondimensional forms as illustrated in Figs. 3a and 3b. The quantity $\phi$ can be normalized by the value of AC voltage of the exposed electrode, $\phi(t)$, in equation (12). Equation (8) can then be solved by imposing a constant boundary condition equal to unity at the upper electrode. Once the dimensionless $\phi$ distribution is determined, the dimensional $\phi$ values at any given time can be obtained by multiplying this distribution with the corresponding value of $\phi(t)$ given by equation (12). Similarly, Eq. (10) can be solved only once at the beginning of computations by using the dimensionless equation for the charge density distribution, which is normalized by $\rho_{c}^{\max } f(t)$. This implies that the boundary condition for the dimensionless charge density on the embedded electrode 
is equal to unity. Once the solution for the dimensionless charge density is established, the dimensional values at any time can be calculated from this distribution by multiplying it with the corresponding value of $\rho_{c}^{\max } f(t)$.

This modeling approach for plasma actuators is implemented in the GHOST code developed at University of Kentucky. GHOST is a pressure-based code based on SIMPLE algorithm with second order accuracy in both time and space. This code is capable of handling complex geometries, moving and overset grids, and includes multiprocessor computation capability using MPI. The overset grid capability of the code enables incorporation of

$$
\begin{array}{lll}
\phi(t)=\phi^{\max } f(t) & \nabla .\left(\varepsilon_{r} \nabla \phi^{*}\right)=0 & \nabla \cdot\left(\varepsilon_{r} \nabla \rho_{c}^{*}\right)=\rho_{c}^{*} / \lambda_{d}^{2} \\
\rho_{c}(t)=\rho_{c}^{\max } f(t) & \phi^{*}=\phi / \phi(t) & \rho_{c}^{*}=\frac{\rho_{c}}{\rho_{c}^{\max } f(t)}
\end{array}
$$

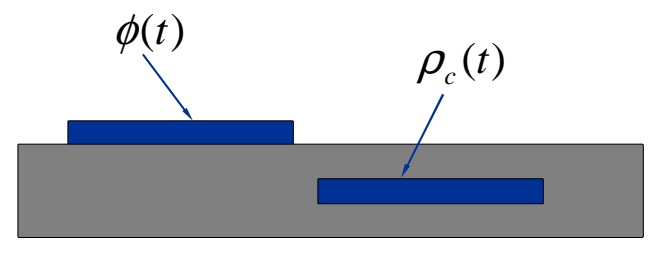

(a) Dimensional variables

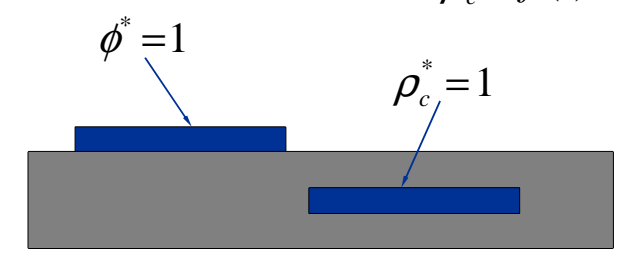

(b) Nondimensional forms of Eqs. (8) and (10)

Figure 3. Dimensional and nondimensional variables and nondimensional form for Eqs. (8) and (10).

plasma actuators into the computations with relative ease since electrodes can be defined as individual solid blocks. The domain can be divided into two separate computational domains: one for the air side and the other for the dielectric wall. The GHOST code has been previously validated against a wide range of test cases and flow conditions and has been used extensively in several low pressure turbine related publications[20,21].

\section{Model Calibration and Validation Using Quiescent Flow Experiments}

In order to calibrate the maximum charge density on the wall, $\rho_{c}^{\max }$, appearing in the model we employed the quiescent flow experiments (Ref. 22) conducted using a single pair of electrodes to better isolate the effects of the actuator on the surrounding air. The details of the actuator geometry and experimental set up are given in Reference [15]. The actuator consists of two $10 \mathrm{~mm}$ wide, $0.102 \mathrm{~mm}$ thick conductive copper strips as electrodes which are separated by a $0.127 \mathrm{~mm}$ thick Kapton dielectric with a $\varepsilon_{r}$ value of 2.7 . Streamwise spacing of electrodes is $0.5 \mathrm{~mm}$.

In the experiments the lower electrode was grounded and plasma region was generated using a square wave with frequency of, $\omega=4.5 \mathrm{kHz}$ and amplitude of $\phi^{\max }=5 \mathrm{kV}$. It should be noted that the experimental data is preliminary and it is used here only to demonstrate the proof of concept for the modeling approach. In the computations our aim was to match the maximum velocity observed in the experiments as well as the experimentally observed flow pattern shown in Figure 4a. From the experiment it was observed that the flow was drawn into the surface region above the embedded electrode by the plasma induced body force. This resulted in a jet issuing to the right of the actuator with a maximum velocity of approximately $1 \mathrm{~m} / \mathrm{s}$.

Based on the flow pattern and maximum velocity criteria, $\rho_{c}^{\max }=0.00750 \mathrm{C} / \mathrm{m}^{3}$ seems to agree well with the experiment. The streamlines obtained from the computation using these values are shown in Figure (4b). The computed flow pattern compares favorably with the experimental flowfield shown in Figure (4a). The computed maximum velocity is $1 \mathrm{~m} / \mathrm{s}$ also matching the experimental value. It should be noted that the boundary layer obtained by the computation appears to be thinner than the experimental data. 


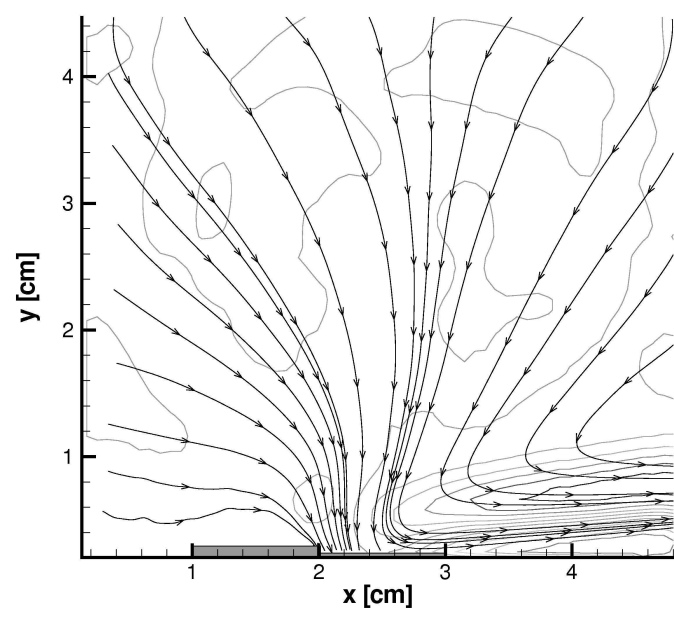

(a) Experiment[22]

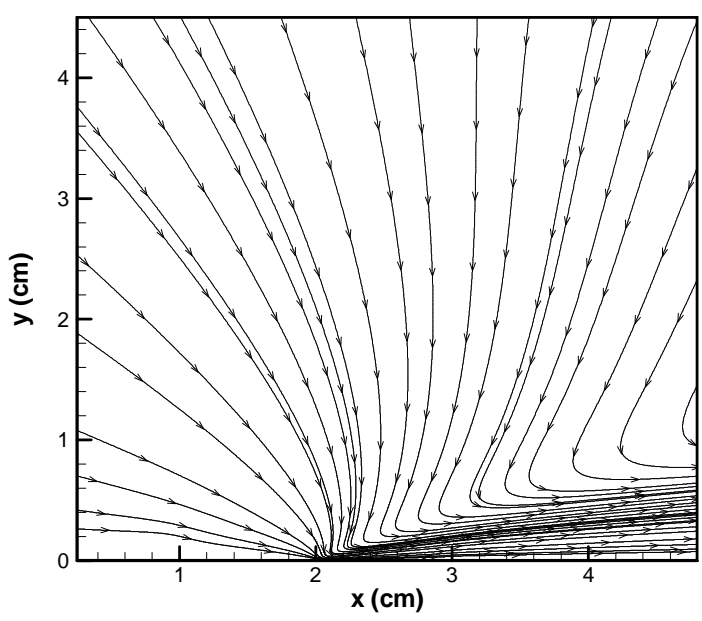

(b) Computation

Figure 4. Comparison of experimental[22] and computed streamlines for the plasma actuator in quiescent flow (The actuator interface is located at the $2 \mathrm{~cm}$ tick.)

The computed electric potential distribution in the vicinity of the electrodes obtained from equation (8) is shown in Figure 5 along with the streamlines of the actuator induced flow. The computed electric potentials show that the strongest electric potential variation, or the electric field, is in the region between the two electrodes. This is also the region where the strongest concentration of plasma is observed in the experiments. The streamlines indicate that the flow is pulled from above into this region and jetted to the right direction as observed experimentally.

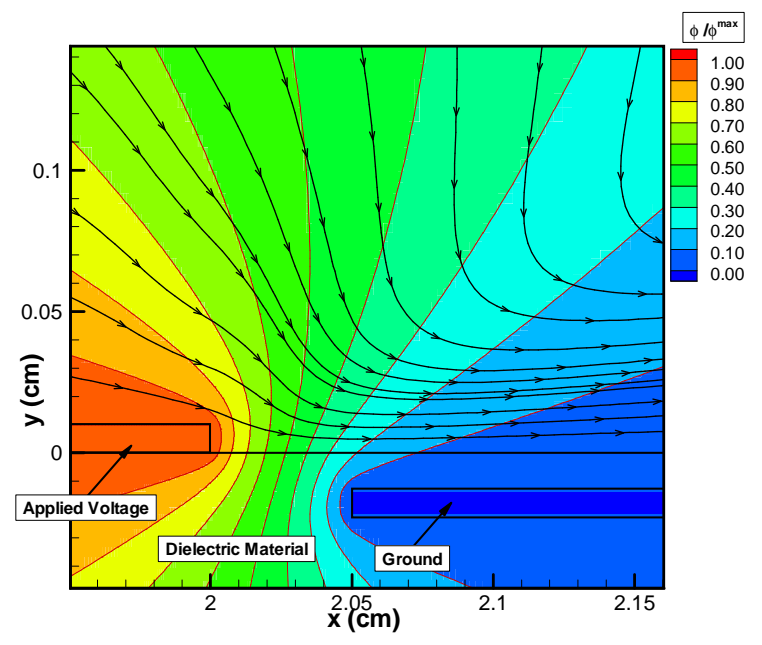

Figure 5. Computed electric potential contours and streamlines in the vicinity of the electrodes

The computed charge density distribution obtained from solution of Eq. (10) is shown in Figure 7 on the air side. It must be noted that this charge density distribution over the surface is obtained without any explicit specification of a charge density variation. Using the embedded electrode as a source for Eq. (10) results automatically in the charge density distribution shown in Figure 7, eliminating any need for explicit specification of a charge density distribution. This aspect of the model is important since it makes the model applicable to any generic electrode shape or orientation without the need of explicit prescription of a charge density distribution.

The magnitude of the resulting body force computed from Eq. (11) is shown in Figure 8. The maximum body force is concentrated in the region over the upper left corner of the embedded electrode where the electric field is the strongest. This is consistent with the experimental observations. 


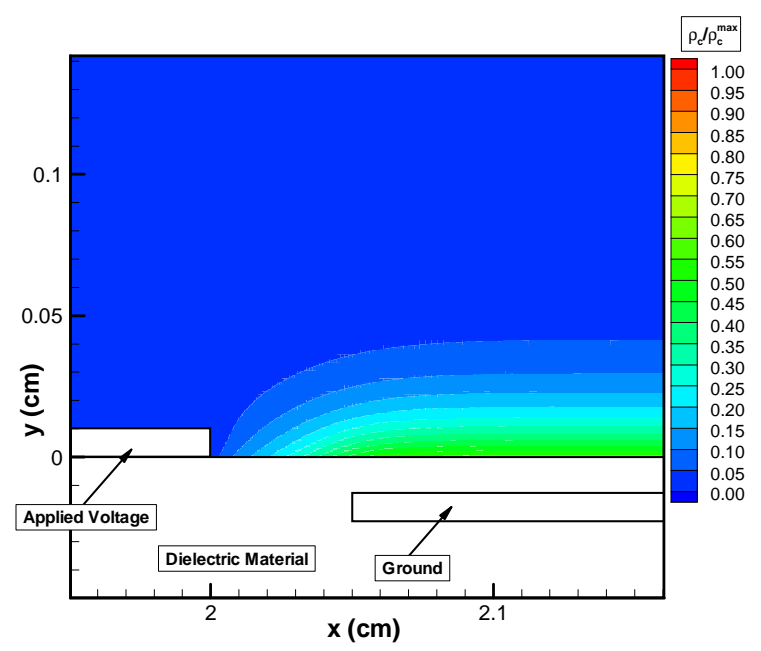

Figure 7. Computed charge density contours in the vicinity of the electrodes

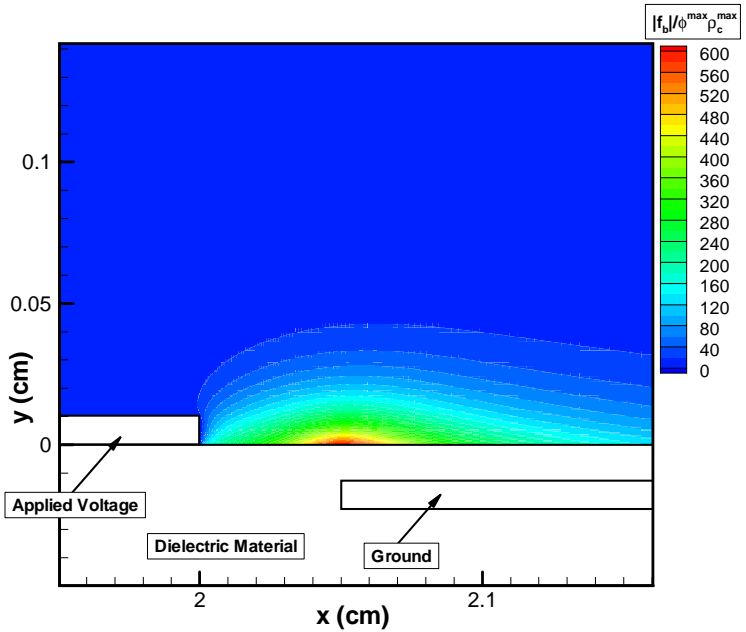

Figure 8. Computed body force magnitude contours in the vicinity of the electrodes

These results show that the modeling technique developed can reproduce the effects of plasma actuators observed in experiments and the approach is promising in computation of plasma flow control applications involving generic actuator geometries and orientations.

\section{Concluding Remarks}

A recently developed phenomenological model to simulate flow control applications using plasma actuators has been further developed and improved in order to expand its applicability to complicated actuator geometries. The new model eliminates the requirement of an empirical charge density distribution shape by using the embedded electrode as a source for the charge density. The resulting model is validated against a flat plate experiment with quiescent environment. The modeling approach incorporates the effect of the plasma actuators on the external flow into Navier Stokes computations as a body force vector which is obtained as a product of the net charge density and the electric field. The model solves the Maxwell equation to obtain the electric field due to the applied AC voltage at the electrodes and an additional equation for the charge density distribution representing the plasma density. These two equations need to be solved only once before the computation. The new modeling approach solves the charge density equation in the computational domain assuming the embedded electrode as a source therefore automatically generating a charge density distribution over the embedded electrode. This makes the model applicable to any generic electrode shape or orientation without the need of explicit prescription of a charge density distribution.

\section{Acknowledgments}

This work is supported by North Dakota NASA EPSCoR through NASA grant NCC3-582. This work is also supported by NASA-Glenn Research Center under Cooperative Agreement NCC3-1040.

\section{References}

${ }^{1}$ Huang, J., Corke, T.C., Thomas, F.O., 2003, "Plasma Actuators for Separation Control of Low Pressure Turbine Blades,"AIAA-2003-1027, AIAA 41 ${ }^{\text {st }}$ Aerospace Sciences Meeting and Exhibit, Reno, NV, January 2003.

${ }^{2}$ Roth, J.R., Sherman, D.M., Wilkinson, S.R., 2000, "Electrohydrodynamic Flow Control with a Glow-Discharge Surface Plasma," AIAA Journal, Vol. 38, No. 7, July 2000.

${ }^{3}$ Corke, T., Cavalieri, D., Matlis, E., 2001, "Boundary Layer Instability on a Sharp Cone at Mach 3.5 with Controlled Input," AIAA Journal, Vol. 40, No 5., pp. 1015.

${ }^{4}$ Corke, T., Jumper, E., Post, M., Orlov, D., McLaughlin, T., 2002, “Application of Weakly ionized Plasmas as Wing Flow Control Devices," AIAA-2002-0350.

5 Post, M.L., Corke, T., 2003, "Separation Control on High Angle of Attack Airfoil Using Plasma Actuators," AIAA $2003-1024$.

${ }^{6}$ Hultgren, L.S., Ashpis, D.E., 2003, "Demonstration of Separation Delay with Glow-Discharge Plasma Actuators," AIAA2003-1025. Reno, NV, Jan. 2003. 
${ }^{7}$ Jacob, J., Rivir, R., Campbell, C., Estevedoreal, J., 2004, "Boundary Layer Flow Control Using AC Discharge Plasma Actuators." AIAA-2004-2128, $2^{\text {nd }}$ Flow Control Conference, Portland, OR.

${ }^{8}$ Post, M.L., Corke, T., 2004, "Separation Control Using Plasma Actuators- Stationary \& Oscillating Airfoils," AIAA-20040841.

${ }^{9}$ Morris, S.C., Corke, T.C., VanNess, D., Stephens, J., Douville, T., “Tip Clearance Control Using Plasma Actuators,”, AIAA2005-0782, January 2005.

${ }^{10}$ Shyy, W. Jayaraman, B., Andersson, A., "Modeling of Glow Discharge-Induced Fluid Dynamics," Journal of Applied Physics, Vol. 92, No. 11, pp. 6434-6443, December 2002.

${ }^{11}$ Hall, K.D., Jumper, E.J., Corke, T.C., McLaughlin, T.E., "Potential Flow Model of a Plasma Actuator as a Lift Enhancement Device," AIAA-2005-0783, January 2005.

${ }^{12}$ Orlov, D.M., Corke, T.C., "Numerical Simulation of Aerodynamic Plasma Actuator Effects," AIAA 2005-1083.

${ }^{13}$ Roy, S., Gaitonde, D., "Radio Frequency Induced Ionized Collisional Flow Model for Application at Atmospheric Pressures,", Journal of Applied Physics, Vol. 96, No. 5, pp. 2476-2481, September 2004.

${ }^{14}$ Roy, S., Gaitonde, D., "Modeling Surface Discharge Effects of Atmospheric RF on Gas Flow Control," AIAA-20050160, January 2005.

${ }^{15}$ Suzen, Y.B., Huang, P.G., Jacob, J.D., Ashpis, D.E., "Numerical Simulations of Plasma Based Flow Control Applications,: AIAA-2005-4633, June 2005, Toronto, Ontario.

${ }^{16}$ Enloe, C.L., McLaughlin, T.E., VanDyken, R.D., Kachner, K.D., Jumper, E.J., Corke, T.C., Post, M., Haddad, O., 2004, "Mechanisms and Responses of a Single Dielectric Barrier Plasma Actuator: Geometric Effects," AIAA Journal, Vol. 42, No. 3, pp. 595-604, March 2004.

${ }^{17}$ Patankar, N. A., Hu, H.H., "Numerical Simulation of Electroosmotic Flow," Analytical Chemistry, Vol. 70, No. 9, pp. 18701881, May, 1998.

${ }^{18}$ Enloe, C.L., Mclaughlin, T.E., Font, G.I., Baughn, J.W., "Parameterization of Temporal Structure in a Single Dielectric Barrier Aerodynamic Plasma Actuator," AIAA-2005-0564, January 2005.

${ }^{19}$ Enloe, C.L., Mclaughlin, T.E., VanDyken, R.D., Fischer, J.C., "Plasma Structure in the Aerodynamic Plasma Actuator," AIAA-2004-0844, January 2004.

${ }^{20}$ Suzen, Y.B., Huang, P.G., "Numerical Simulation of Unsteady Wake/Blade Interactions in Low Pressure Turbine Flows Using an Intermittency Transport Equation," Journal of Turbomachinery, Vol. 127, No. 3, pp. 431-444, July 2005.

${ }^{21}$ Suzen, Y.B., Huang, P.G., "Comprehensive Validation of an Intermittency Transport Model for Transitional Low-Pressure Turbine Flows,” The Aeronautical Journal, Vol. 109, No. 1093, pp. 101-118, March 2005.

${ }^{22}$ Jacob, J.D., Ramakumar, K., Anthony, R., Rivir, R.B., "Control of Laminar and Turbulent Shear Flows Using Plasma Actuators," Fourth International Symposium on Turbulence and Shear Flow Phenomena, TSFP-4, Williamsburg, VA, June 2005. 